\title{
Diagnostic and management problems in a complex case of connective tissue disease
}

\author{
SS Yeap, CM Deighton, RJ Powell, RC Read, RG Finch
}

\begin{abstract}
Summary
A 28-year-old Nigerian woman presented with persistent pyrexia, marked pruritis, eosinophilia, myalgias, flitting arthralgias, serositis and massive splenomegaly. Intensive investigation for an infective or neoplastic aetiology proved negative. Empirical treatment for helminthic infections and tuberculosis was unhelpful. Although there were no specific clues to suggest an underlying connective tissue disease, a trial of steriods and azathioprine was introduced, with no obvious response. Her condition deteriorated to a point where it was decided that intravenous immunosuppressive therapy was needed and subsequently, her condition improved remarkably. This patient illustrates the problems in the diagnosis and management of complex disorders, particularly when classical tests for connective tissue diseases are absent. Also, we would like to report that marked pruritis can be associated with connective tissue disease
\end{abstract}

Keywords: connective tissue disease, pyrexia, pruritis, eosinophilia, immunosuppression

\section{Case report}

City Hospital, Nottingham, NG5 1PB, UK

Department of

Rheumatology

SS Yeap

CM Deighton

Department of

Infectious Diseases

RJ Powell

Clinical Immunology Unit, Department of Immunology, Queens Medical Centre,

University Hospital

NHS Trust,

Nottingham, NG7

2UH, UK

RC Read

RG Finch

Correspondence to

Dr SS Yeap, Department of Rheumatology, Leicester

Royal Infirmary NHS Trust,

Leicester LE1 5WW, UK

Accepted 1 June 1995
A 28-year-old Nigerian woman presented in September 1992 with a 10-day history of profound malaise, fever, rigors, sore throat, productive cough, loose stools, low back pain, flitting myalgia and arthralgia, intense pruritus and an evanescent rash. She had left Nigeria seven years previously. She had had a normal vaginal delivery three months prior to admission. There was no significant other previous medical history. She was noted to have a variable pyrexia (up to $39.2^{\circ}$ ), tender inguinal lymphadenopathy, tachycardia, dyspnoea, a soft systolic murmur at the apex, flitting synovitis affecting the knees, wrists and ankles, and an evanescent diffuse papular rash associated with marked pruritus. A Schrimer's tear test was normal. Two months after initial presentation, the above clinical state persisted but in addition, she developed cervical lymphadenopathy, palpable splenomegaly, and splinter haemorrhages.

Investigations revealed a haemoglobin of $10.3 \mathrm{~g} / 1$ (normochromic, normocytic), negative Coomb's tests, esoinophilia peaking at $3.27 \times$
$10^{9} / 1$, erythrocyte sedimentation rate $95 \mathrm{~mm} / \mathrm{h}$, C-reactive protein maximum value $403 \mathrm{mg} / \mathrm{l}$ (normal $<10 \mathrm{mg} / \mathrm{l}$ ), normal liver, renal and bone biochemistry, creatine kinase normal, lactate dehydrogenase $2350 \mathrm{U} / 1$ (230-460), iron $8 \mu \mathrm{mol} / 1 \quad(1-30)$, total iron-binding capacity $30 \mu \mathrm{mol} / 1(45-72)$, ferritin 92500 (17-165) and angiotensin-converting enzyme $75 \mathrm{U} / 1$ (8-52). An infectious disease screen revealed the following negative or normal results: numerous blood, stool and urine cultures, Mantoux test, Monospot test, antistreptolysin O titre, serology for syphilis, coxiella, malaria, filaria, schistosomiasis, strongyloidiasis, brucellosis, leishmaniasis, toxoplasmosis, toxocariasis, amoebiasis, fungal precipitins, Lyme disease, hydatid disease and viral titres, including parvovirus, HIV and HTLV-1.

Immunological investigations showed a negative autoantibody screen, including ENA, DNA and ANCA, polyclonal elevations in IgA $6.04 \mathrm{~g} / 1$ (1.25-4.25), IgG $20.7 \mathrm{~g} / 1$ (5-16), IgE $764 \mathrm{kU} / 1$ (1.53-114), with normal IgM and serum electrophoresis and normal C3 and C4 with a markedly elevated $\mathrm{C} 3 \mathrm{~d}, 20 \mathrm{U} / \mathrm{ml}(5-12)$. Radiology demonstrated an initially normal chest X-ray and computed tomography (CT) scan confirmed the enlarged spleen, thymus and heart. Repeated echocardiograms showed a small persistent posterior pericardial effusion, but no evidence of endocarditis. Biopsy of skin showed fibrinoid necrosis in superficial dermal papillae with oedema. Muscle biopsy showed no evidence of vasculitis. Bone marrow demonstrated florid reactive hyperplasia with eosinophilia, but no evidence of tuberculosis. A liver biopsy and subsequent surgical samples of spleen and lymph nodes showed non-specific inflammatory changes with no evidence of tuberculosis, lymphoma or granulomata.

On her first admission, a Trichomonas and Candida vaginal infection had been treated with no improvement to her fever. Empiricial trials of amoxycillin, erythromycin and metronidazole were likewise unhelpful. In view of the esoinophila and pruritus, a trial of thiabendazole was given, with no benefit. She was commenced on prednisolone $40 \mathrm{mg}$ and azathioprine $150 \mathrm{mg}$ daily for presumed connective tissue disease, again with no benefit. This was therefore stopped and followed by a longterm trial of antituberculous therapy (isoniazid, rifampicin and ethambutol) which also resulted in no improvement.

Her intense pruritus continued throughout 
her illness and remained resistant to antipruritic therapy consisting of antihistamines and chlorpromazine. After five months of malaise, fevers, pruritus and arthralgias, she became acutely unwell and breathless. She had been persistently anaemic, (haemoglobin $7.1 \mathrm{~g} / \mathrm{dl}$ ) and investigations did not support blood loss or haemolysis. The splenomegaly had increased, with an edge palpable at the umbilicus. She had a large right-sided pleural effusion and large pericardial effusion. Blood-stained fluid from the pleural effusion was negative on cluture and cytology. All other investigations were unchanged from previously, although her rheumatoid factor was now positive at 1/10 240. It was decided to perform a splenectomy, because of the recurrent anaemia, and the lack of a tissue diagnosis. However, histology of the spleen showed non-specific inflammatory changes only, with no evidence of tuberculosis, lymphoma or granulomata.

Postoperatively she remained extremely unwell, being tachypnoeic with profound arthralgias. Frank synovitis of her left wrist developed which was aspiration negative on culture, and X-ray showed a small erosion on the ulnar styloid. It was decided to commence her on weekly boluses of cyclophosphamide, starting at $500 \mathrm{mg}$, increasing to $1 \mathrm{~g}$, with initially $60 \mathrm{mg}$ of prednisolone. After the third bolus, her condition had improved markedly. The pleural and pericardial effusions gradually resolved, and the arthralgias and synovitis abated. She is currently maintained on $7 \mathrm{mg}$ of prednisolone daily, with oral boluses of $500 \mathrm{mg}$ of oral cyclophosphamide every four weeks. She still has polyarthralgia with mild synovitis at the wrists, but excellent joint function.

\section{Discussion}

This patient illustrates the management difficulties of complex multisystem disorders. Connective tissue disease is said to account for $8-13 \%$ of all causes of a pyrexia of unknown origin. ${ }^{1,2}$ The initial concern was that she had an infectious disease, but multiple repeated investigations were negative and trials of empirical antimicrobial chemotherapy were unsuccessful in resolving her problems. We were concerned about an underlying neoplastic process, but the investigations tended to diminish these concerns. We considered an inflammatory connective tissue disease, though initial investigations, for example, ANA, were unhelpful and the introduction of prednisolone (albeit only at a modest dose) and azathioprine did not appear to improve her condition.

1 Petersdorf RG, Beeson PB. Fever of undetermined origin report on 100 cases. Medicine 1961; 40: 1-30.

2 Larson EB, Featherstone HJ, Petersdorf RG. Fever of undetermined origin: diagnosis and follow-up of 105 cases, 1970-80. Medicine 1982; 61: 269-92.

3 Weller PF. Eosinophilic syndromes. In: Wyngaarden JB, Smith LH Jr, Bennet JC, eds Cecil textbook of medicine, 19th edn. Philadelphia: WB Saunders, 1992; p 966.

4 Arnett FC, Edworthy SM, Bloch DA, et al. The ARA 1987 Arnett FC, Edworthy SM, Bloch DA, et al. The ARA 1987
revised criteria for the classification of rheumatoid arthritis. revised criteria for the classification

5 Ohta A, Yamaguchi M, Kaneoka H, Nagayoshi T, Hida M Adult Still's disease: review of 228 cases from the literature. f Rheumatol 1987; 14: 1139-46.
Because of the lingering doubt that she could have an underlying infectious disease, immunosuppression was delayed until her condition deteriorated rapidly and markedly. High-dose cyclophosphamide was then introduced with rapid response, and has kept her well with no further hospital admissions for disabling symptoms or life-threatening complications.

Even with the benefit of hindsight, there are still unanswered questions in this patient. It remains difficult to fit her into a diagnostic category. Eosinophilia is associated with a number of idiopathic inflammatory connective tissue diseases, including polyarteritis nodosa/ polyangiitis, allergic granulomatosis with angiitis (Churg-Strauss syndrome), eosinophilic fasciitis and severe rheumatoid arthritis with or without Felty's syndrome. ${ }^{3}$ The first three diagnoses were unlikely in view of the negative ANCA, the lack of pulmonary signs throughout the course of her disease, and negative tissue biopsies, respectively. Although she had a positive rheumatoid factor and an erosion on X-ray, she had no prolonged, symmetrical small joint synovitis to fulfil the 1987 revised criteria for the diagnosis of rheumatoid arthritis. ${ }^{4}$ With the arthralgia and fever, adultonset Still's disease was a possibility. Eosinophilia has been described in a minority of Japanese patients ${ }^{5}$ and a very high ferritin level considered diagnostically helpful when other causes have been excluded. ${ }^{6}$ However, the typical macular non-pruritic rash present in $89 \%$ of adult patients was not observed ${ }^{5}$ and the severe pruritus described by our patient is not characteristic. A further possible multisystem disease is that of idiopathic hypereosinophilic syndrome, but our patient did not have the criteria required namely, sustained hypereosinophilia for more than six months. ${ }^{7}$ In fact, the hypereosinophilia resolved in our patient before the introduction of corticosteriods or immunosuppression. Finally, the intense pruritus experienced by our patient has not been previously described in other patients with connective tissue disease and is not a recognised causes of pruritus in the standard medical textbooks. ${ }^{8}$

In conclusion, marked pruritus can be a feature of connective tissue disease. Our patient also illustrates the dilemmas in the management of a severe multisystem disease, the inadequacies of our classification systems for idiopathic complex connective disease, and the power of non-specific, blind, high-dose immunosuppressives when all else had failed.

6 Ohta A. Serum ferritin can be useful for diagnosis in adul onset Still's disease. (letter). F Rheumatol 1989; 16: 413.

7 Fauci AS, Harley JB, Roberts WC, Ferrans VJ, Gralnick HR, Bjornson BH. The idiopathic hypereosinophilic syndrome. Clinical, pathophysiologic and therapeutic considerations. Ann Intern Med 1982; 97: 78-92.

8 Kennedy CTC. Skin disease. In: Souhami RL, Moxham J, eds, Textbook of medicine, Edinburgh: Churchill Livingstone, 1990; p 1148 